\title{
On-Line Diagnosis and Energy Assessment of Centrifugal Pumps under a Thermodynamic Approach Method
}

\author{
Eduar R. Rivera' ${ }^{1}$, Guillermo E. Valencia ${ }^{2}$ and Jorge E. Duarte ${ }^{3 *}$ \\ 'Department, Aguas de Cartagena, Cartagena, Colombia; eduarrivera@mail.uniatlantico.edu.co \\ 2Department of Mechanical Engineering, KAI Research Group, Universidad del Atlántico, Barranquilla, \\ Colombia; guillermoevalencia@mail.uniatlantico.edu.co \\ ${ }^{3}$ Department of Mechanical Engineering, DIMER Research Group, Universidad \\ del Atlántico, Barranquilla, Colombia; jorgeduarte@mail.uniatlantico.edu.co
}

\begin{abstract}
Background/Objectives: The implementation of a novel method for the characterization of energy efficiency in centrifugal pumps based on the first law of thermodynamics is studied, with which the energy consumption of the turbomachine can be diagnosed. Methods: With the measurement of temperature and pressure at the inlet and outlet of the pump, energy efficiency is determined, without the need to measure electrical or mechanical parameters, which allows determining quickly on site if the pump is operated in an area of high efficiency. Findings: The results allow corroborating the high predictive capacity of the proposed methodology so that the trend is followed compared with efficiency data calculated by conventional methods. A deviation close to $1 \%$ was observed in the average efficiencies, compared with the developed method. Application: To develop an online diagnosis and energy assessment of Centrifugal Pumps based on the thermodynamic Approach Method.
\end{abstract}

Keywords: Centrifugal Pumps, Energy Assessment, Energy Efficiency, On-Line Diagnosis, Thermodynamic Approach

\section{Introduction}

The potential of numerical simulation in hydraulic turbomachinery lies, on the one hand, in the prediction of the operating conditions and, on the other hand, in the analysis of the internal flow, something that from the experimental point of view is difficult or impossible to achieve. A great advantage over the measures is the availability of the equations of the time evolution of the flow. Also, the performance of parametric studies, for example, variations in geometry and loading conditions, is quick and cheap.

The Euler 3D equations allow calculating with certain precision the flow in a turbomachine, even though in that the effects of the friction are not negligible $\underline{1}$. For the calculation of the three- dimensional flow in hydraulic turbomachines considering the effects of friction, the Navier-Stokes equations must be considered, which must be averaged temporarily following the Reynolds procedure if the turbulence of the flow is considered 2 .

Compare CFD and energy characterization methods for radial pumps and do it for axial ones ${ }^{3,4}$. Investigate a pump-turbine with CFD, and other authors such as perform a statistical characterization for the study of rot dynamic pumps $\frac{5,6}{6}$. The secondary flow in the stationary coupled calculation is investigated by considers the influence of the rotor on the optimization of the spiral housing $\frac{7.8}{}$. Analyze both the potential interaction and the non-stationary effect of the rotation speed difference between the rotor and a warped radial diffuser ${ }^{9}$. In recent years, numerical investigations have been carried out using turbulence models.

Who used the model $\mathrm{k}-\varepsilon$ and the model SST (Shear Stress Transport)? This author in addition to making several fundamental observations on the theoretical predictive capacity of turbulence models, recommend using 
at least turbulence models of two equations for use in turbomachinery ${ }^{10}$. A diagnostic methodology of centrifugal pumps is applied but focused from the energy point of view is addressed in this research. For this, an analysis of data of measurements in real time was considered, which led to a model for the prediction of efficiency from a novel thermodynamic approach, which is compared by traditional efficiency estimation methods in this type of turbomachinery.

\section{Methodology}

This section presents a detailed description of the general purpose of the software/hardware solution developed, the presentation of the technical aspects of the experimental configuration developed and finally the fundamental equations and the thermodynamic model implemented for the diagnosis.

\subsection{General Aim of the Software/Hardware Solution}

The main purpose of the tool developed is to develop an in-situ diagnosis of the energy efficiency and flow of centrifugal pumps, by applying the first principle of thermodynamics from the pressure and temperature conditions of the fluid at the pump inlet and outlet, without measuring electrical and mechanical parameters. Another, the advantage of the development over traditional techniques is that the thermodynamic method is much simpler regarding instrumentation. Since to determine the electromechanical efficiency of the pump motor assembly, it does not require the mea- surement of the electrical power supplied to the motor, for the estimation of the pump efficiency, delivered flow, electric motor efficiency and the overall efficiency of the system.

The developed user interface allows saving, in addition to the records of temperatures and suction and discharge pressures, the flow rates delivered by the pump and power consumed by the drive motor by direct measurement. The application allows graphing the fluid inlet and outlet temperatures, to perform statistical calculations of the central trend measurement, to determine the degree of dispersion of the data, allowing comparative studies between the efficiency and flow estimated with both methods, if the necessary measuring instruments are available.

\subsection{Experimental Equipment}

Fluid conditions, were measured using high-precision PT100 Omni grad T TST310 temperature probes from ENDRESS HAUSER (A) with a TMT 180 temperature transducer from ENDRESS HAUSER (B) in a range of 0 to $150^{\circ} \mathrm{C}$ and Danfoss MBS 33 (C) pressure sensors, one absolute pressure from 0 to 6 bar to measure the suction pressure, and the other 0 to 16 bar to measure the discharge pressures as shown in Figure 1.

$<$ Insert Figure 1 here $>$

The PLC (D) used to capture the pressure, and temperature data was a Motorola ACE3600 RTU, which receives the 4 to 20 MA signals and uses an algorithm to perform the calculations of the implemented thermodynamic model and then transfer all this data to the computer (E). Both the WEG motor (F) and the KSB Meganorm 80-250
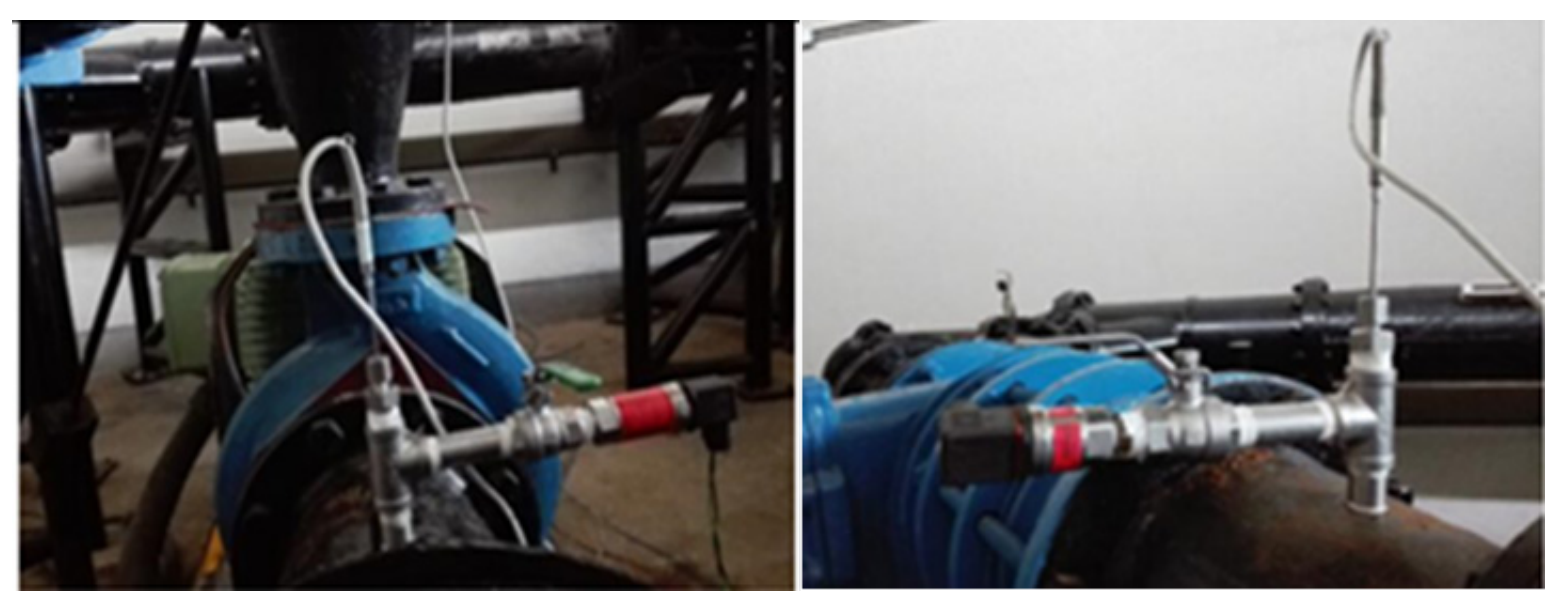

Figure 1. Installation of instrumentation to capture pressure and temperature data. 


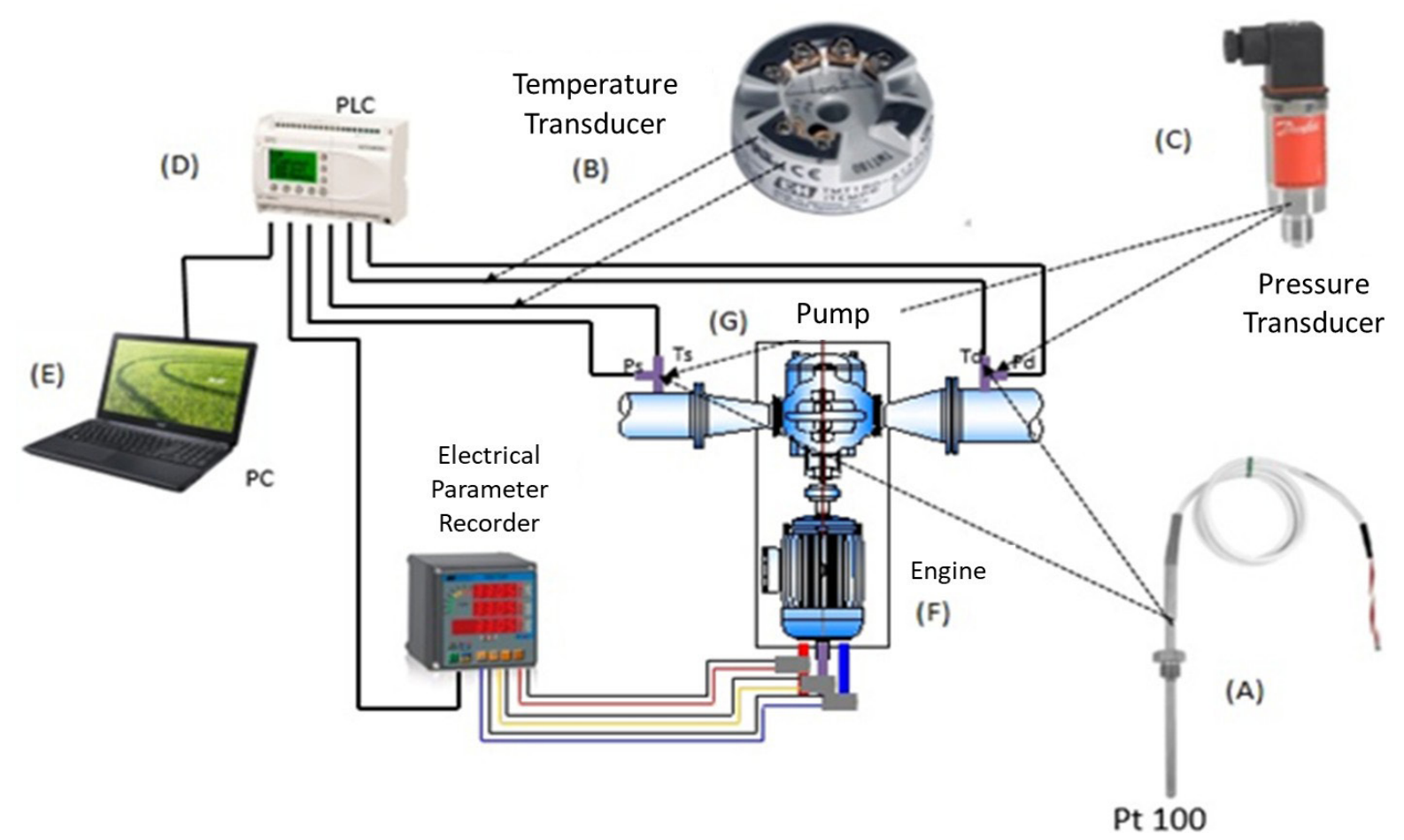

Figure 2. Schematic diagram of process and instrumentation required.

(G) pump wasan instrument to measure the energy consumed by the motor and the flow rate moving the pump. Figure 2 shows a schematic diagram of the configuration made with its respective instrumentation.

\subsection{Fundamental Equations}

In the case of the traditional method, the efficiency of a pump is given by the ratio of the hydraulic power supplied to the fluid by the pump to the power supplied to the pump through the shaft, as expressed in the Equation $(1)^{11}$ :

$$
\eta_{p}=\frac{P_{W}}{P_{p}}
$$

Where $\eta_{p}$ is the efficiency of the pump, $\mathrm{P}_{\mathrm{w}}$ is the hydraulic power and $\mathrm{P}_{\mathrm{p}}$ is the mechanical power supplied to the pump by the motor, which is obtained by means of a dynamometer on the pump shaft. Alternatively, this can be achieved by adjusting the power supplied to the motor by the motor loss factor, while the hydraulic power $\mathrm{P}_{\mathrm{w}}$ is given by Equation (2),

$$
P_{w}=Q \cdot H \cdot \delta
$$

where $\mathrm{Q}$ is the volumetric flow rate in units of volume per unit of time $\mathrm{m}^{3} / \mathrm{s}$, measured by a flow meter at the pump outlet, $\delta$ is the specific weight of water in $\mathrm{kN} / \mathrm{m}^{3}$ and $\mathrm{H}$ is the increase in pressure through the pump, which is obtained by applying Bernoulli between the pump inlet and outlet and obtaining equations 3 and 4 for horizontal and vertical pumps respectively:

$$
\begin{aligned}
& H=z_{d-} z_{s}+h_{g d-} h_{g s}+\frac{v_{d}^{2}-v_{s}^{2}}{2 g} \\
& H=z_{d-} z_{w}+h_{g d}+\frac{v_{d}^{2}}{2 g}
\end{aligned}
$$

where $Z_{s}$, is the potential energy of the liquid given by the relative elevation from the reference line to the center of the pump suction manometer, $Z_{w}$ is the distance of the reference line from the surface of the liquid, $Z_{d}$ is the potential energy of the liquid given by the relative elevation from the reference line to the center of the pump discharge manometer, $h_{v d}$ is the manometric pressure recorded by a manometer at the pump discharge, $h_{v s}$ is the manometric pressure at the pump discharge.

$$
h_{v s}=\frac{v_{s}^{2}}{2 \mathrm{~g}}
$$




$$
h_{v d}=\frac{v_{d}^{2}}{2 \mathrm{~g}}
$$

where $\mathrm{V}_{\mathrm{s}}$ and $\mathrm{V}_{\mathrm{d}}$ are the suction and discharge velocities of the pump respectively and $g$ is the gravitational constant. Finally, the overall efficiency of the system is given by Equation (7)

$$
\eta_{\mathrm{oa}}=\frac{p_{w}}{p_{\text {mot }}}
$$

where $\eta_{\mathrm{oa}}$ is the hydraulic efficiency of the pump motor assembly, $\mathrm{Pw}$ is the hydraulic power calculated by equation 2 and $\mathrm{P}_{\text {mot }}$ is the electrical power supplied to the motor.

The above equations, used to determine the efficiency of a pump correspond to ANSI/HI 1.6-2000 and 2 standards. 6-2000, which are designed to be applied in laboratories, and can be used in the field making some adjustments, however, not having dynamometers to measure the power on the $\mathrm{P}_{\mathrm{p}}$ axis, the product obtained is the overall efficiency $\eta_{\text {oa }}$, or electromechanical efficiency of the pump motor assembly, leaving the uncertainty about what is the real efficiency of the pump, and what is the efficiency of the motor.

Using the thermodynamic method, which results from the principle of energy conservation, it is based on the measurement of the fluid conditions at the pump inlet and outlet points 1 and 2 as shown in Figure 3. Considering the changes in enthalpy, kinetic and gravitational energy, the actual energy transmitted by the pump to the fluid has been determined by variations in pressure, temperature, fluid velocity and differences in height between the pressure measuring instruments compared to a common reference plane ${ }^{12}$. This method does not require flow measurement to determine the efficiency, which is the main difference with the traditional method ${ }^{13}$.

The hydraulic efficiency of a pump can be calculated with the thermodynamic method from Equation (8)

$$
\eta_{\mathrm{p}}=\frac{E_{h}}{E_{m+E_{x}}}
$$

where $\eta_{p}$ is the hydraulic efficiency of the pump, $E_{h}$ is the change of total energy per unit mass of the working fluid in an ideal isentropic process between input and output conditions, Em is the change of energy per unit mass supplied to the fluid by the pump, and Ex is the external losses that do not manifest themselves in an increase in

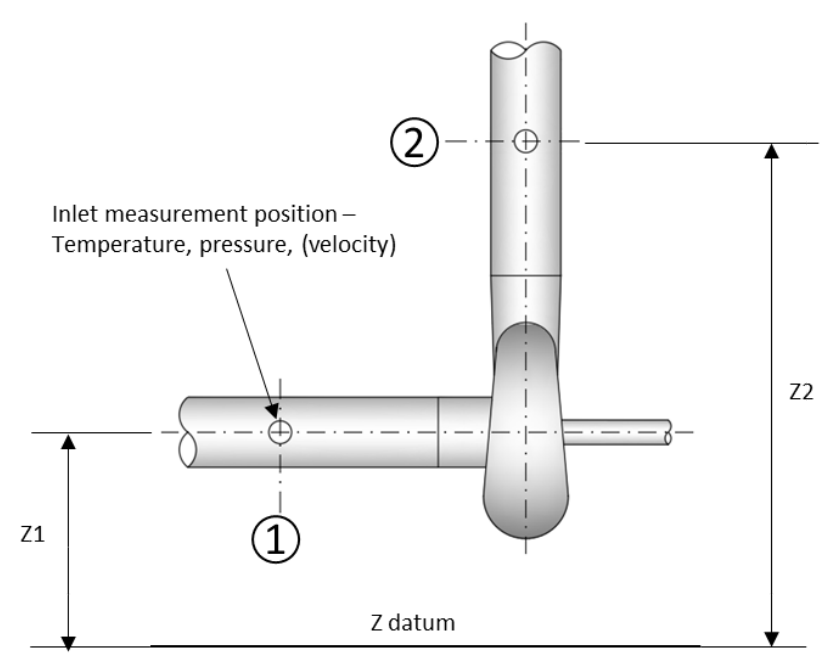

Figure 3. Measuring positions and reference plane.

water temperature, which occurs basically in bearings and seals (12).

The terms $E_{h}, E_{m y} E_{x \text { equation } 8 \text { can be calculated using }}$ the Equations (9), (10) and (11)

$E_{h}=V\left(h_{g d}-h_{g s}\right)+\left(v_{d}^{2}-v_{s}^{2}\right) / 2+g\left(z_{d}-z_{s}\right)$,

$E_{m}=a\left(h_{g d}-h_{g s}\right)+C_{P}\left(D_{2}-D_{1}\right)+\frac{v_{d}^{2}-v_{s}^{2}}{2}+g\left(z_{d}-z_{s}\right)+\Delta E m$

$E_{x}=\frac{P_{x}}{q_{m}}$

where $h_{g d}$ and $h_{g s}$ are the enthalpies of the fluid in the discharge and inlet of the pump, $V_{d}$ and $V_{s}$ are the fluid velocities at the discharge and inlet of the pump, $D_{2}$ and $\mathrm{D}_{1}$ represent the coefficient of isothermal expansion, $C_{P}$ heat capacity of the fluid at constant pressure, $\Delta \mathrm{Em}$ is the term for correction by heat transfer that is not absorbed by the fluid, $\mathrm{q}_{\mathrm{m}}$ mass flow and $\mathrm{P}_{\mathrm{x}}$ are the losses in bearings and seals.

From the hydraulic and mechanical energies per unit mass transferred by the pump to the fluid, excluding the $\mathrm{E}_{\mathrm{x}}$ term of equation 8 corresponding to the external losses that do not affect the fluid temperature, ignoring the kinetic and potential terms in Equations (9) and (10), Equation (12) is obtained, which allows efficiency to be obtained from the first principle of thermodynamics. 


$$
\eta_{\mathrm{p}}=\frac{1}{1+\frac{C_{p} \cdot\left(\mathrm{D}_{2}-\mathrm{D}_{1}\right)}{g \cdot\left(\hat{h}_{g d^{-}}-h_{g s}\right)}}
$$

The flow rate delivered by the pump can be calculated from Equation (13) by measuring the power absorbed by the motor, the efficiency of the pump and the motor ${ }^{14}$.

$\mathrm{Q}=\frac{P_{\mathrm{mot}} \cdot \eta_{\mathrm{p}} \cdot \eta_{\mathrm{mot}}}{\rho g H}$

where the engine efficiency is estimated from the load factor $\frac{14}{14}$ using the Equation (14):

$$
\text { load factor }=\frac{I_{\text {logged }}}{I_{\text {nominal }}} \cdot \frac{V_{\text {logged }}}{V_{\text {nominal }}}
$$

where $\mathrm{I}_{\text {logged }}$ is the current consumed by the motor, $\mathrm{I}_{\text {nominal }}$ is the motor plate current, $\mathrm{V}_{\text {logged }}$ is the motor supply voltage and $V_{\text {nominal }}$ is the motor plate voltage.

\section{Result and Discussion}

The objective of the pumping station test was to validate the efficiency of the thermodynamic method at different operating pressures and to construct the pump curve to compare it with the original curve provided by the manufacturer in order to validate the results obtained in the 5 tests at different pump discharge pressures of 110, 100, 95 and $92 \mathrm{MCA}$.

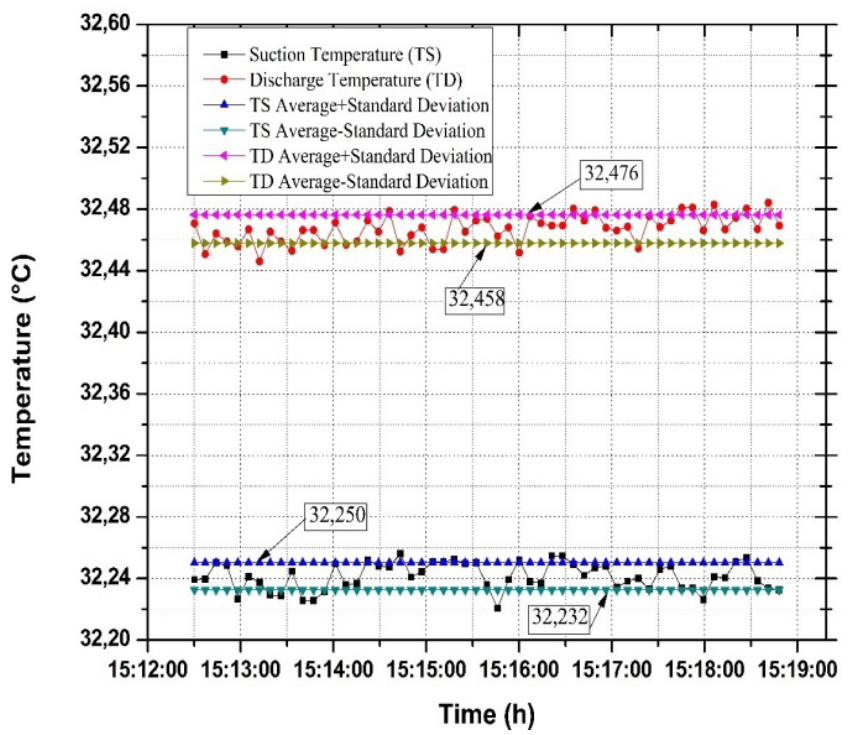

\subsection{Suction, Discharge and Flow Temperatures for $100 \mathrm{MCA}$}

Figure 4(a) shows the dispersion of the recorded suction and discharge temperature data, where a maximum suction temperature of $32.25^{\circ} \mathrm{C}$, a minimum of $32.22^{\circ} \mathrm{C}$, and an average of $32.24^{\circ} \mathrm{C}$ were obtained, while for discharge temperatures the maximum was $32.48{ }^{\circ} \mathrm{C}$, the minimum $32.44{ }^{\circ} \mathrm{C}$ and the average $32.46{ }^{\circ} \mathrm{C}$, which means that the temperature increase of the fluid was very little due to the high levels of efficiency of the pump according to the energy balance of the pump. In order to evaluate the degree of variability of the measured data, the graph shows for both the suction and discharge temperatures two horizontal lines, which represent the average value of all temperatures plus the standard deviation, and the same average value minus the standard deviation, obtaining that for this test almost the entire point cloud for both variables are located between these lines, which shows the good degree of uncertainty of the measurement.

Figure 4(b) shows the dispersion of the flow rate data calculated from Equation (3), taking the calculated thermodynamic efficiency, the motor plate efficiency and the electrical power consumed by the motor, recorded by the net analyzer used during the test, the flow rate calculations gave a maximum, a minimum and an average of 170,144 and $157 \mathrm{~m}^{3} /$ hour respectively.

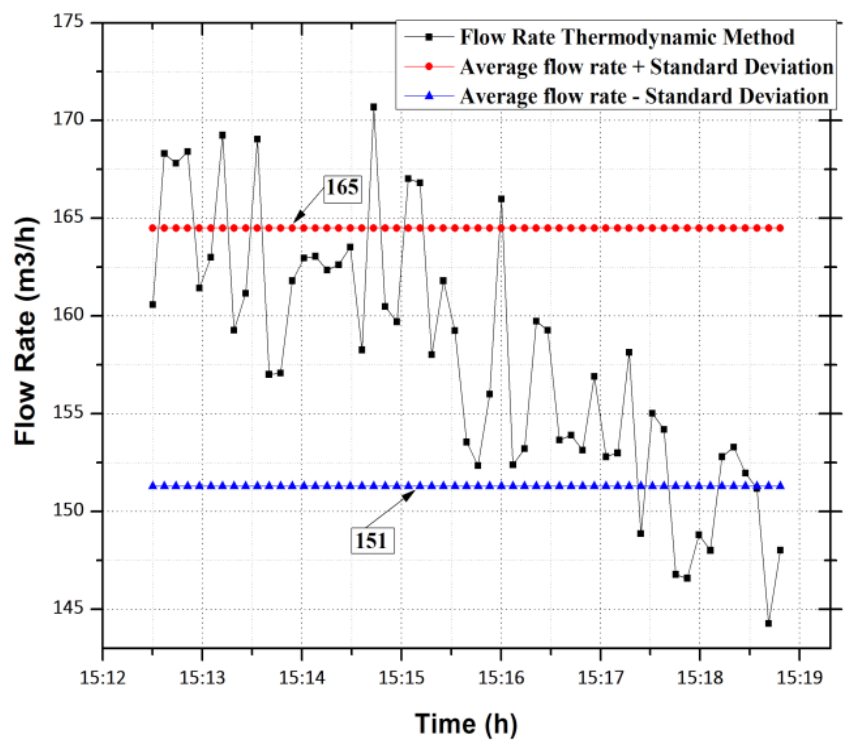

Figure 4. 100 MCA pressure test, a) suction temperatures, b) flow rate by thermodynamic method. 
The graph shows in addition to the flow rate results, two horizontal lines, which represent the average value of all the flow points plus the standard deviation, and the same average value minus the standard deviation, the above to show the degree of dispersion of the flow rate data, in the graph there is a downward trend in the flow rate this is due to the fact that the pressure of the test could not be stabilized and this increase of 100 MCA at the beginning of the test.

\subsection{Pump Efficiency for 100 MCA}

As for the pump efficiency behavior for both methods for a discharge pressure of $100 \mathrm{MCA}$, the dispersion of the efficiency data calculated by the thermodynamic method from the temperature records has to be calculated, the maximum, minimum and average efficiencies found were $55 \%$ and $49 \%$ respectively as shown in Figure 5(a). Similarly, the efficiency results show two horizontal lines, which represent the average value of all efficiency points and the standard deviation, and the same average value minus the standard deviation, to show the degree of dispersion of the efficiency data and the degree of repeatability and confidence level of the experiment. Also, Figure 5(b) presents a comparison between efficiency calculated by the thermodynamic method and the traditional method, where it is shown that the efficiency calculated by the traditional method

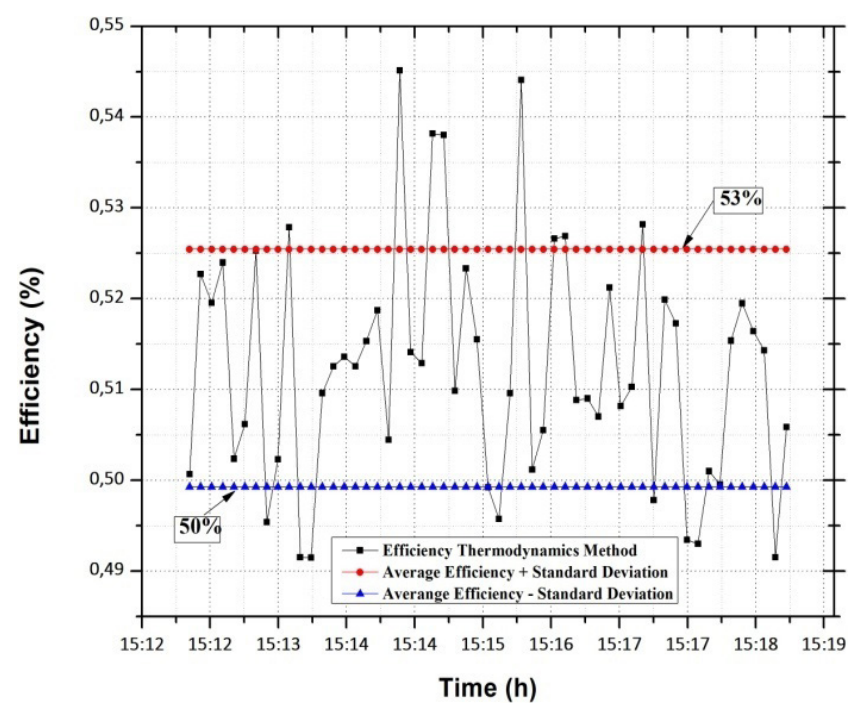

Figure 5. Efficiency performance for $100 \mathrm{MCA}$ pressure. (a) Thermodynamic method. (b) comparison between traditional and thermodynamic methods.

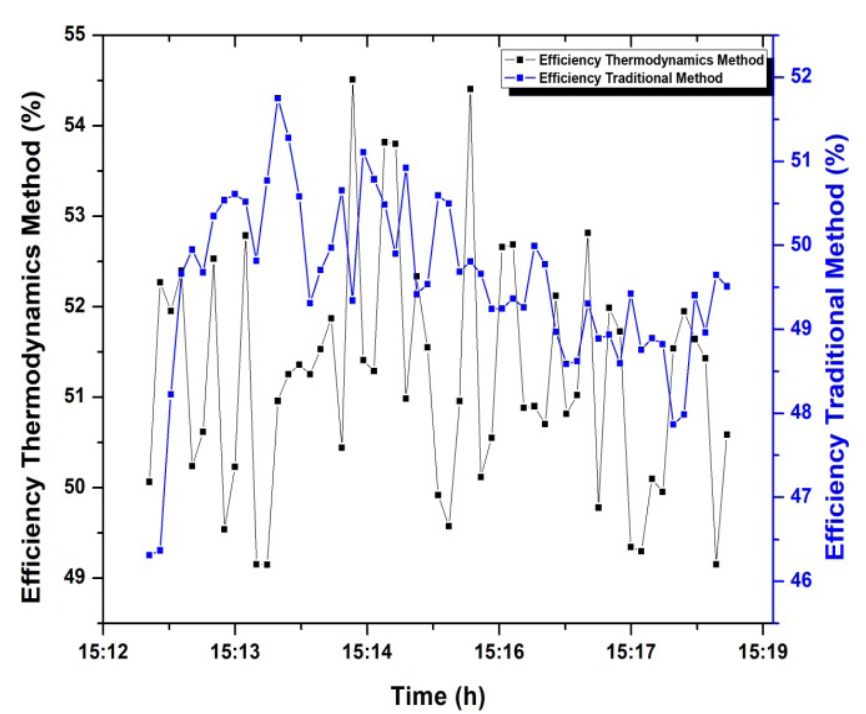

is slightly below the point cloud of the thermodynamic method.

\subsection{Comparison of flow rate results for 100 MCA}

Figure 6(a) shows a comparison between the flow rate calculated by the thermodynamic method and the flow rate recorded by direct measurement through the flowmeter used during the test, where it can be seen that the point cloud of the flow rate recorded on site by direct measurement is slightly below the cloud of points corresponding to the flow rate calculated by the thermodynamic method.

The procedure described above was repeated for different head values, which are summarized in the following figure:

Table 1 shows that the deviations presented are in the order of 9 thousandths of a degree ${ }^{\circ} \mathrm{C}$, for suction and discharge temperatures, $1 \%$ for efficiency and $6.61 \mathrm{~m}^{3} / \mathrm{h}$ for flow, results are aligned with the literature consulted in the sense that at higher pressures the results present less error.

\section{Conclusions}

It is possible to measure the efficiency and flow rate of a centrifugal pump by means of the thermodynamic method operating with discharge pressures below 

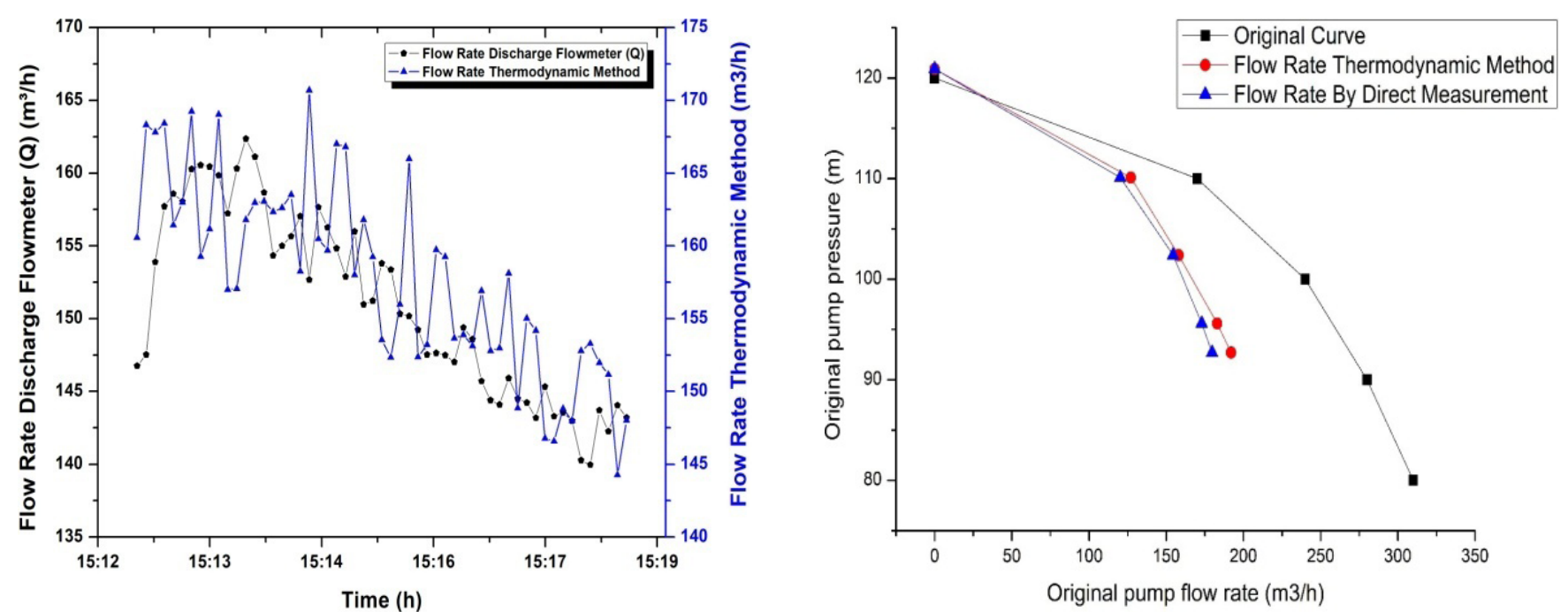

Figure 6. Comparison of method response at 100 MCA. (a) Flow rates. (b) Pump characteristic curve.

Table 1. Central trend measurements and efficiency and flow calculations for $100 \mathrm{MCA}$

\begin{tabular}{|l|c|c|c|c|c|}
\hline & $\begin{array}{c}\text { Temp_Suction } \\
\text { TS }\end{array}$ & $\begin{array}{c}\text { Temp_Discharge } \\
\text { TD }\end{array}$ & Efficiency & Flow Rate & Pressure \\
\hline Average & $32.241^{\circ} \mathrm{C}$ & $32.467^{\circ} \mathrm{C}$ & $51 \%$ & $157.90 \mathrm{~m}^{3} / \mathrm{hr}$ & $102 \mathrm{MCA}$ \\
\hline Max & $32.256^{\circ} \mathrm{C}$ & $32.484^{\circ} \mathrm{C}$ & $55 \%$ & $170.69 \mathrm{~m}^{3} / \mathrm{hr}$ & $105 \mathrm{MCA}$ \\
\hline Mín & $32.221^{\circ} \mathrm{C}$ & $32.446^{\circ} \mathrm{C}$ & $49 \%$ & $144.27 \mathrm{~m}^{3} / \mathrm{hr}^{2}$ & $100 \mathrm{MCA}$ \\
\hline Standard Deviation & 0.009 & 0.009 & $1 \%$ & 6.61 & 1.54 \\
\hline Median & 32.241 & 32.468 & $51 \%$ & 158.13 & 102.32 \\
\hline
\end{tabular}

100MCA, obtaining more reliable results if several measurements are made and an average data is taken for the calculation of efficiency, in order to filter the variability that occurs in temperature measurements when they are made at pressures below 100MCA.

The flow rate delivered by the pump was measured with a good degree of precision, based on the calculated thermodynamic efficiency, taking the actual data of the power absorbed by the motor using specialized sensors and estimating its efficiency from the plate data and adjusting it by means of the motor load factor. Also, the use of computer tools such as PLCs and software allowed the programming of online data acquisition and analysis, facilitating the real-time estimation of the efficiency and flow rate delivered by the pump.

Finally, the comparisons between the data obtained by traditional and thermodynamic methods showed great proximity in the results obtained, which gives validity to this tool for in situ determination of efficiency and flow rate.

\section{Acknowledgment}

Aguas de Cartagena supported this research with E. Rivera, and the Engineering Department of Universidad del Atlántico with the Efficient Energy Management Research Group Kai, with G. Valencia, and by the DIMER Research Group, with J. Duarte, both from Mechanical Engineering Program.

\section{References}

1. Hagelstein D, Hillewaert K, Van den Braembussche RA, Engeda A, Keiper R, RautenbergM. Experimental and numerical investigation of the flow in a centrifugal compressor volute. Journal of Turbomachinery. 2000; 122(1):22-31. Crossref.

2. Govatsos PA, Papantonis DE. A characteristic based method for the calculation of three-dimensional incompressible, turbulent and steady flows in hydraulic turbomachines and installations. International Journal for Numerical Methods in Fluids. 2000; 34(1):1-30. Crossref. 
3. Pavesi G, Cavazzini G. Rotor-stator interactions in a radial flow pump. 7th Conference on Turbo machinery, Fluid Dynamics and Thermodynamics. 2007. p. 1-11.

4. Numerical and experimental investigation of tank-type axial-flow pump device. Available from: Crossref.

5. Muggli FA, Holbein P, Dupont P. CFD calculation of a mixed flow pump characteristic from shutoff to maximum flow. Journal of Fluids Engineering. 2002; 124(3):798-802. Crossref.

6. Camargo C, García C, Duarte J, Rincón A. Modelo estadístico para la caracterización y optimización en bombas periféricas. Ingeniería y Desarrollo. 2018; 36(1):18-39. Crossref.

7. Kreiselpumpe. Available from: Crossref.

8. Majidi K, Siekmann HE. Numerical calculation of secondary flow in pump volute and circular casings using 3D viscous flow techniques. International Journal of Rotating Machinery. 2000; 6(4):245-52. Crossref.
9. Shi F, Tsukamoto H. Numerical study of pressure fluctuations caused by impeller-diffuser interaction in a diffuser pump stage. Journal of Fluids Engineering. 2001; 123(3):466-74. Crossref.

10. Treutz G. Numerische Simulation der instationärenStrömung in einer Kreiselpump. Doctoral dissertation; Berlin. 2002. p. 1-122.

11. American National Standard Institute. Available from: https://en.wikipedia.org/wiki/American_National_ Standards_Institute

12. Thorne EW, Neal AN. Pump efficiency testing by the thermodynamic method-an independent view. Journal of Power and Energy. 2000; 214(3):255-68. Crossref.

13. Papa F, Radulj D, Karney B, Robertson M. Pump energy efficiency field testing and benchmarking in Canada. Journal of Water Supply: Research and Technology-Aqua. 2014; 63(7):570-7. Crossref.

14. Kaya D, Yagmur EA, Yigit KS, Kilic FC, Eren AS, Celik C. Energy efficiency in pumps. Energy Conversion and Management. 2008; 49(6):1662-73. Crossref. 